\title{
CARATHÉODORY MEASURE AND A GENERALIZATION OF THE GAUSS-GREEN LEMMA $\dagger$
}

\author{
BY \\ JOHN F. RANDOLPH
}

\section{INTRODUCTION}

1. The Gauss-Green lemma for the plane connects the double integral of a partial derivative of a function over a region $R$ with the line integral of the function around the curve $C$ bounding $R$. This connection is given by the formula

$$
\iint_{R} \frac{\partial f(x, y)}{\partial y} d x d y=\int_{C} f(x, y) \cos \beta d s
$$

where $s$ is the arc length of $C$ and $\beta$ is the angle made by the external normal to $C$ at a variable point of $C$, with the positive $y$-axis. Thus for the line integral to have a meaning it is necessary that $C$ have length and consequently be defined by two functions $x=\phi(t), y=\psi(t)$ of bounded variation in $t$.

2. The applications of this lemma, through Green's theorem, are numerous. Consequently many investigations have been concerned with the types of regions and boundaries for which the lemma is valid. In most cases it has been deemed inherent in such a relation that the boundary be a curve, i.e., that an order relation be known among the points of the boundary. However, attacking the problem by radically different methods, J. P. Schauder $\ddagger$ obtained results for a class of boundaries with no order relation prescribed.True, the only boundaries shown by Schauder to be admissible are those represented by two functions each satisfying the Lipschitz conditions, thus indirectly again introducing order and also a condition more restricting than that of bounded variation.

Also Schauder assumed that all points of the boundary of the second class $\S$ project on the $x$-axis in a set of Lebesgue measure zero. Thus so simple a region as all points of the unit circle except the points $0 \leqq x<1$ of the $x$-axis does not satisfy his conditions.

$\dagger$ Presented to the Society, October 27, 1934; received by the editors January 8, 1935.

$\ddagger$ Fundamenta Mathematicae, vol. 8 (1926), p. 1. Schauder states his results in terms of an integral over a volume and an integral over the boundary of the volume, but, as he points out, analogous results hold connecting $n$ - and $(n-1)$-dimensional integrals, $n=2,3, \cdots$ We discuss his results for $n=2$.

$\S$ This subset of the boundary is defined in $\$ 16$ below. 
3. The present paper contains a proof of the Gauss-Green lemma under what seems extreme simplification of the conditions on the boundary. For a simply connected region there is no restriction except that the boundary have Carathéodory linear measure finite. Then by methods which have the effect of the usual crosscut scheme, applicable regions are extended to a wide class not simply connected. Furthermore, simplicity of restrictions on boundaries is gained by more careful analysis of properties of boundaries, and not by specialization of the function $f(x, y)$.

From the proofs given, analogous results are seen to hold connecting $n$ and $(n-1)$-dimensional integrals.

\section{Carathéodory measure of a SET AND its Closed subSets}

4. Carathéodory $\dagger$ developed on five axioms a general theory of measure in which most of the theorems of the usual Lebesgue theory have analogues. The theorem that the inner measure of a set is the upper limit of the measures of closed subsets of the set, which plays such a central role in the Lebesgue theory, has not, however, been shown to follow from Carathéodory's five axioms. This closed subset theorem does follow, as proved by Hahn, $\ddagger$ if in place of Carathéodory's fifth axiom the following modification is used:

Aхіом $\mathrm{V}^{\prime}$. To each point set $A$ there is a sequence of open sets whose intersection contains $A$ and has the same measure as the outer measure of $A$.

After developing his general theory of measure, by merely postulating the existence of a number associated with each set, Carathéodory gave the following specific method of attaching a number to a set.

Let $A$ be an arbitrary set in a euclidean space $R_{q}$ of $q$ dimensions. With $\rho$ a positive number let $U_{1}, U_{2}, \cdots$ be a sequence of convex sets open in the space $R_{q}, \S$ each with diameter less than $\rho$, whose union contains $A$. With $d_{k}$ the diameter of $U_{k}$, consider the sums

$$
d_{1}+d_{2}+\cdots
$$

for all such sequences of point sets. Designate the least upper bound, which may be $+\infty$, of such sums by $L_{\rho} A$. Then $L_{\rho} A$ does not decrease as $\rho$ decreases. Thus as $\rho \rightarrow 0, L_{\rho} A$ approaches a limit, finite or infinite, which in either case is called the exterior linear measure of $A$ and is represented by $L^{*} A$.

The exterior two-dimensional measure of $A$ is also defined by means of sets $U_{1}, U_{2}, \cdots$, each with diameter less than $\rho$, except that $d_{k}$ is replaced

$\dagger$ Göttinger Nachrichten, 1914, p. 404.

$\ddagger$ Hahn, Theorie der reellen Funktionen, vol. 1, 1921, Theorem III, p. 445.

$\S$ Carathéodory did not assume the sets $U_{k}$ to be open, but proved that the same number would be obtained if open convex sets were used. 
by the two-dimensional diameter of $U_{k}$. The two-dimensional diameter of an open convex set is the least upper bound of the Lebesgue plane measures of the projections of the set on planes of all possible orientations.

Exterior linear measure is shown by Carathéodory to satisfy his five measure axioms. In proving that exterior linear measure satisfies his fifth axiom, Carathéodory did not use the fact that each $U_{k}$ is open, but merely that it is linearly measurable. Upon following this proof, but using in addition the openness of $U_{k}$, one will see that Hahn's axiom $\mathrm{V}^{\prime}$ is also satisfied. It thus follows from the above reference to Hahn, that if a set $A$ has inner linear measure $L_{*} A$ finite, there is a sequence of closed subsets of $A$ whose union has the same linear measure as the inner linear measure of $A$. This closed subset theorem plays a fundamental role in the work that follows.

5. One easily sees the following projection relation:

If $A$ is a plane set and $l$ a line of the plane, the projection $A_{l}$ of $A$ on $l$ has Lebesgue exterior measure $m^{*} A_{l}$ less than or equal to $L^{*} A$.

With this projection relation established, Gross $\dagger$ proved that if each point of $A_{l}$ is the image of at least $N$ points of $A$ then $m^{*} A_{l} \leqq L^{*} A / N$. Specifically, if $L^{*} A$ is finite and each point of $A_{l}$ is the image of an infinite number of points of $A$ then $m^{*} A_{l}=0$.

It follows also from the above projection relation and the closed subset theorem that, if $A$ is linearly measurable with LA finite, then $A_{l}$ is Lebesgue measurable.

\section{Measurable subsets of the union of a Sequence of Closed Sets}

6. A line perpendicular to the $x$-axis through a point of a plane set $B$ may or may not contain a lowest point of $B$ on it. We designate the set of all such lowest points, when they exist, by $B^{1}$. In general we designate by $B^{m}$ the set of all points $p$ of $B$ such that exactly $m-1$ points of $B$ lie below $p$. on the same perpendicular to the $x$-axis.

Again a line perpendicular to the $x$-axis through a point of $B$ may contain a finite or an infinite number of points of $B$. We designate by $\mathscr{B}^{m}$ (or $\left.\mathcal{B}^{\infty}\right)$ the collection of all points of $B$ on all lines perpendicular to the $x$-axis that contain exactly $m$ (or an infinite number of) points of $B$.

Arguments similar to those of Schauder show that if $B$ is a Souslin set, each subset of $B$ defined above is Carathéodory linearly measurable. Corresponding theorems of course hold if $B$ is the union of a sequence of closed

$\dagger$ Monatshefte für Mathematik und Physik, vol. 29 (1918), pp. 174-176. 
sets. However, simpler proofs can be made for the more restricted sets and at the same time furnish all that is necessary for our purpose. $\dagger$

7. Suppose then $B$ is the union of a sequence of closed sets $K_{1}, K_{2}, \cdots$.

Toward establishing the measurability of the subsets of $B$ mentioned above we notice that the set $P(B)$, of all points on all lines perpendicular to the $x$-axis through points of $B$, is linearly measurable. For $P(B)=P\left(K_{1}\right)$ $+P\left(K_{2}\right)+\cdots$ and each $P\left(K_{i}\right)$ is linearly measurable, since the part of it in or on any square is closed.

With $k$ a positive integer let $W_{k}^{h}$ be the collection of all points of the plane whose $y$-coordinates satisfy the relation $h / 2^{k} \leqq y<(h+1) / 2^{k}, h=1,2, \cdots$. Since each set $W_{k}^{h}$, as well as $B$, is the union of a sequence of closed sets, the same is true of their intersection. Consequently, from the above proof, the set $P\left(B W_{k}^{h}\right)$ is linearly measurable.

Let $h_{1}<h_{2}<\cdots<h_{m}$ be $m$ integers. We define

$$
P^{h_{1} h_{2} \cdots h_{m}}
$$

as all points of all lines perpendicular to the $x$-axis that contain points of $B$ in each of the strips

$$
W_{k}^{h_{1}}, W_{k}^{h_{2}}, \cdots, W_{k}^{h_{m}}
$$

but no point of $B$ in any strip below $y=\left(h_{m}+1\right) / 2^{k}$, other than these.

The point set (1) is linearly measurable. For the set of lines perpendicular to the $x$-axis with points of $B$ in all the strips (2) may be written as

$$
G_{1}=\prod_{i=1}^{m} P\left(B W_{k}^{h_{i}}\right) .
$$

Then to obtain the set (1) we must remove from $G_{1}$ any line perpendicular to the $x$-axis that contains a point of $B$ outside the strips (2), but still below $y=\left(h_{m}+1\right) / 2^{k}$, i.e.,

$$
G_{2}=\sum_{j=-\infty}^{h_{1}-1} P\left(B W_{k}^{j}\right)+\sum_{j=h_{1}+1}^{h_{2}-1} P\left(B W_{k}^{j}\right)+\cdots+\sum_{j=h_{m-1}+1}^{h_{m}-1} P\left(B W_{k}^{j}\right) .
$$

Since each point set $P\left(B W_{k}^{j}\right)$ is linearly measurable, the point sets $G_{1}$ and $G_{2}$

$\dagger$ Schauder used a general measure $\Phi$ satisfying Carathéodory's axioms I-IV and a modification (different from Hahn's) of axiom V. He did not however prove the closed subset theorem for his general measure. To obtain this theorem he introduced a specific measure $\Phi_{0}$, a modification of one defined by Gross (loc. cit.), which satisfies his modified axioms. In his proof he used some measure properties of $B^{m}$ which in turn he obtained from measure properties of Souslin sets. For Carathéodory linear measure, indeed for the general measure satisfying Hahn's modification of the five axioms, the closed subset theorem followed without the use of Souslin sets and we now establish the requisite measure properties of $B^{m}$. 
are linearly measurable. Consequently, $P^{h_{1} h_{2}} \cdots h_{m}=G_{1}-G_{1} G_{2}$ is also linearly measurable.

We now define the set

$$
B_{k}^{h_{1} h_{2} \cdots h_{m}}
$$

as all points $p$ of $B$ in the strip $W_{k}^{h_{m}}$ such that a line through $p$ perpendicular to the $x$-axis contains points of $B$ in all of the strips (2), but no point of $B$ in any strip below $y=\left(h_{m}+1\right) / 2^{k}$ other than these. The formulation for this set, $B_{k}^{h_{1} \ldots h_{m}}=\left(B W_{k}^{h_{m}}\right) P^{h_{1}} \cdots h_{m}$, shows it to be linearly measurable, a fact we use in proving

TheOREM 1. The subset $B^{m}$ of $B$ is linearly measurable.

First consider the set

$$
E_{k}=\sum B_{k}^{h_{1} \cdots h_{m}}+\sum B_{k+1}^{h_{1} \cdots h_{m}}+\cdots+\sum B_{k+j}^{h_{1} \cdots h_{m}}+\cdots,
$$

where each summation sign means the union of the sets indicated as the $m$ distinct integers $h_{1}<h_{2}<\cdots<h_{m}$ take on all possible values. The set $E_{k}$ is then the union of a countable number of linearly measurable sets, so is itself linearly measurable.

We next assert that $B^{m} \subset E_{k}$. For if $p$ is a point of $B^{m}$ there are exactly $m-1$ other points of $B$ below $p$ on the same perpendicular to the $x$-axis. Then with $k$ fixed, there is a number $j$ so large that the distance between any two of these $m$ points is greater than $1 / 2^{k+j}$. These $m$ points then belong to some set of $m$ strips $W_{k+j}^{h_{1}}, \cdots, W_{k+j}^{h_{m}}$, and there are no points of $B$ on their common line in any strip below $y=\left(h_{m}+1\right) / 2^{k+i}$ other than these. Thus $p$ belongs to some set $B_{k+j}^{h_{1} \ldots h_{m}}$ and so to $E_{k}$.

Thus the set $E=E_{1} \cdot E_{2} \ldots$ is linearly measurable and also $B^{m} \subset E$.

We assert, conversely, that $B^{m} \supset E$. For if $p$ were a point of $E$ and there were less than $m-1$ points of $B$ below $p$ on the same perpendicular to the $x$-axis, $p$ and these points would not lie in any $m$ distinct strips, so $p$ would not belong to any set $B_{k}^{h_{1} \cdots h_{m}}$, so not to $E$. However, if there were more than $m-1$ points of $B$ below $p$, for every $k$ large enough, say $k>K$, there would be more than $m$ strips including $p$ and the points of $B$ below $p$. Thus $p$ would not belong to any set $B_{k}^{h_{1} \ldots h_{m}}, k>K$, so not to $E_{k}, k>K$, and finally not to $E$.

Thus $B^{m}$ is the linearly measurable set $E$.

8. Toward proving $\mathbb{B}^{m}$ linearly measurable we designate by

$$
\mathbb{B}_{k}^{h_{1} h_{2} \cdots h_{m}}
$$

the totality of all points $p$ of $B$ such that the line through $p$ perpendicular 
to the $x$-axis contains points of $B$ in each of the strips $W_{k}^{h_{1}}, \cdots, W_{k}^{h_{m}}$, but no others. This set is then the part of $B$ on those lines $P^{h_{1}} \cdots h_{m}$ that do not contain points of $B$ above $y=\left(h_{m}+1\right) / 2^{k}$; that is,

$$
\mathscr{B}_{k}^{h_{1} \cdots h_{m}}=B\left[P^{h_{1} \cdots h_{m}}-P^{h_{1} \cdots h_{m}} \sum_{j=h_{m}+1}^{\infty} P\left(B W_{k}^{j}\right)\right] .
$$

This formulation shows the set to be linearly measurable.

We now state

TheOREM 2. The subset $\mathbb{B}^{m}$ (and $\left.\mathbb{B}^{\infty}\right)$ of $B$ is linearly measurable.

One will see that the linear measurability of $\mathcal{B}^{m}$ follows from that of $\mathcal{B}_{k}{ }_{k} \cdots h_{m}$ in the same way the linear measurability of $B^{m}$ followed from that of $B_{k}{ }^{h_{1}} \cdots h_{m}$. Then $\mathcal{B}^{\infty}=B-\sum_{m=1}^{\infty} \mathcal{B}^{m}$ is also linearly measurable.

\section{NORMAL SETS AND SIMPLY CONNECTED REGIONS}

9. Following Schauder, a family $F_{A}$ of circles will be said to cover a plane set $A$ if every point of $A$ is the center of a sequence of circles of $F_{A}$ with radii approaching zero. The set $A$ is said to be normal with respect to a measure if in every $F_{A}$ covering $A$ there exists a mutually exclusive sequence of circles whose union contains almost all of $A$. Here a circle includes its circumference.

From the Vitali covering theorem $\dagger$ it follows that every bounded set $A$ is normal with respect to Lebesgue plane measure. It is not known whether under any of the definitions of linear measure, the set $A$ is normal with respect to linear measure even if $A$ is linearly measurable with linear measure finite or, in fact, even if $A$ is closed. The peculiar adaptability of Carathéodory linear measure to our problem is shown by the fact that the boundary $B$ of of every simply connected region is normal with respect to Carathéodory linear measure if $L B$ is finite. It is necessary, however, before proving this fact to obtain several auxiliary results.

10. With $c(p, r)$ a circle with center $p$ and radius $r$, we shall call the limit superior and limit inferior as $r$ approaches zero of the ratio

$$
\frac{L^{*} A c(p, r)}{2 r}
$$

the upper and lower exterior density of $A$ at $p$ and represent them by $\bar{D}^{*}(A, p)$ and $\underline{D}^{*}(A, p)$ respectively. If $A$ is linearly measurable the asterisk is not used and the word "exterior" is dropped.

W. Sierpińskił has shown that in every $F_{A}$ covering $A$ there exists a se-

† Carathéodory, Vorlesungen über Reelle Funktionen, p. 229.

$\ddagger$ Fundamenta Mathematicae, vol. 9, p. 172. 
quence of mutually exclusive circles $c_{1}, c_{2}, \cdots$ such that if $C_{n}$ is a circle (not necessarily belonging to $F_{A}$ ) with the same center, but diameter three times that of $c_{n}$, then the union $S=C_{1}+C_{2}+\cdots$ contains $A$. He also shows that if $L^{*} A$ is finite, $\bar{D}^{*}(A, p) \leqq 1$ at almost all points of $A$. We use these two facts in proving

Lemma 1. If $L^{*} A$ is finite and the lower exterior density of $A$ is bounded from zero by a positive constant $k$ for almost all of $A$, then $A$ is normal. $\dagger$

From the conditions of the lemma and Sierpiński's density theorem, mentioned above, the subset $A^{\prime}$ of $A$ where simultaneously $k \leqq \underline{D}^{*}(A, p)$ and $\bar{D}^{*}(A, p) \leqq 1$ has $L^{*} A^{\prime}=L^{*} A$.

Let $F_{A}$ be a family of circles covering $A$. Then the collection of all circles $c(p, r)$ of $F_{A}$ such that, with $p$ a point of $A^{\prime}$,

$$
\frac{k}{2}<\frac{L^{*} A c(p, r)}{2 r},
$$

while the concentric circle $c(p, 3 r)$, which may not belong to $F_{A}$, is such that

$$
\frac{L^{*} A c(p, 3 r)}{6 r}<2,
$$

is a family $F_{A^{\prime}}^{\prime}$ of circles covering $A^{\prime}$ in the sense defined above.

Then from Sierpinski's covering theorem, there exists in $F_{A^{\prime}}^{\prime}$ a sequence of mutually exclusive circles $c\left(p_{1}, \boldsymbol{r}_{1}\right), c\left(p_{2}, \boldsymbol{r}_{2}\right), \cdots$ such that the union of the larger circles $c\left(p_{1}, 3 r_{1}\right), c\left(p_{2}, 3 r_{2}\right), \cdots$ contains $A^{\prime}$. Since (1) is true for each small circle and these circles are mutually exclusive,

$$
k \sum_{1}^{n} r_{i}<\sum_{1}^{n} L^{*} A c\left(p_{i}, r_{i}\right)=L^{*}\left[A \sum_{1}^{n} c\left(p_{i}, r_{i}\right)\right] \leqq L^{*}\left[A \sum_{1}^{\infty} c\left(p_{i}, r_{i}\right)\right] \leqq L^{*} A .
$$

Thus the series of radii converge and

$$
k \sum_{1}^{\infty} r_{i} \leqq L^{*}\left[A \sum_{1}^{\infty} c\left(p_{i}, r_{i}\right)\right] .
$$

But the union of the larger circles contains $A^{\prime}$, almost all of $A$, and (2) is true for each of these circles, so

$$
L^{*} A \leqq \sum_{1}^{\infty} L^{*} A c\left(p_{i}, 3 r_{i}\right)<12 \sum_{1}^{\infty} r_{i}
$$

From (3) and (4),

† Henceforth we use "normal" for "normal with respect to Carathéodory linear measure." 


$$
k L^{*} A<12 L^{*}\left[A \sum_{1}^{\infty} c\left(p_{i}, r_{i}\right)\right] \text {. }
$$

There is then a number $n$ such that, for $s_{1}=\sum_{1}^{n} c\left(p_{i}, r_{i}\right), k L^{*} A<12 L^{*} A s_{1}$. Thus $(12-k) L^{*} A>12\left(L^{*} A-L^{*} A s_{1}\right)$, so, since $s_{1}$ is linearly measurable (closed),

$$
L^{*}\left(A-A s_{1}\right)<\left(\frac{12-k}{12}\right) L^{*} A
$$

Next let $F_{A^{\prime}}^{\prime \prime}$ denote the collection of all circles of $F_{A^{\prime}}^{\prime}$, that have no point in common with $s_{1}$. Since $s_{1}$ is closed this family covers $A^{\prime}-A^{\prime} s_{1}$. We now proceed as above, but use the set $A^{\prime}-A^{\prime} s_{1}$ instead of $A^{\prime}$ and the family of circles $F_{A^{\prime}}^{\prime \prime}$ instead of $F_{A^{\prime}}^{\prime}$, and obtain a set $s_{2}$ (the union of a finite number of mutually exclusive circles of ${F_{A}^{\prime}}^{\prime \prime}$ ) and a relation similar to (5), namely,

$$
L^{*}\left[\left(A-A s_{1}\right)-\left(A-A s_{1}\right) s_{2}\right]<\left(\frac{12-k}{12}\right) L^{*}\left(A-A s_{1}\right) .
$$

But $s_{2}$ has no point in common with $s_{1}$, so, with the aid of (5),

$$
L^{*}\left[A-A\left(s_{1}+s_{2}\right)\right]<\left(\frac{12-k}{12}\right) L^{*}\left(A-A s_{1}\right)<\left(\frac{12-k}{12}\right)^{2} L^{*} A .
$$

We thus see the existence of a sequence of mutually exclusive sets $s_{1}, s_{2}, \cdots$ each consisting of a finite number of mutually exclusive circles of $F_{A}$, such that for each $n$,

$$
L^{*}\left[A-A\left(s_{1}+s_{2}+\cdots\right)\right] \leqq L^{*}\left[A-A\left(s_{1}+\cdots+s_{n}\right)\right]<\left(\frac{12-k}{12}\right)^{n} L^{*} A .
$$

But $k$ is positive and $L^{*} A$ is finite so $A$ is normal.

11. By a region $R$ we shall mean a connected portion of the plane, and by the boundary $B$ of $R$, all points $b$ such that every circle $c(b, r)$ contains both points of $R$ and points of the complement of $R$. The boundary is a closed point set and is hence linearly measurable. A region is simply connected if every simple closed curve of the region contains on its interior only points of the region.

We now prove the proposition mentioned earlier.

THEOREM 3. The boundary $B$ of a simply connected region $R$ (not the whole plane) is normal if $L B$ is finite.

This theorem follows from the above lemma upon showing that $\underline{D}(B, b) \geqq 1 / 2$ at each point $b$ of $B$. First let $c(b, r)$ be a circle with center $b$ 
which does not contain all of $R$. The circumference of $c(b, r)$ then contains points of $R$, since $R$ is connected, and points of the complement of $R$, since $R$ is simply connected. Consequently there are points of $B$ on this circumference and, moreover, on the circumference of every concentric circle $c\left(b, r^{\prime}\right)$ with $r^{\prime}<r$.

Let $U_{1}, U_{2}, \cdots$ be a sequence of open convex sets whose union contains $B c(b, r)$. If $U_{k}$ contains $b$ let $I_{k}$ be the radius of the smallest circle with center $b$ containing $U_{k}$. If $U_{k}$ does not contain $b$ let $I_{k}$ be the difference of the radii of the two circles with centers $b$, one the smallest containing $U_{k}$, the other the largest containing no point of $U_{k}$. Since $I_{k}$ is not greater than the diameter $d_{k}$ of $U_{k}$ and there is a point of $B$ at every distance $\leqq r$ from $b, r \leqq \sum I_{k} \leqq \sum d_{k}$. Thus $r \leqq L B c(b, r), \dagger$ so

$$
\underline{D}(B, b)=\liminf _{r \rightarrow \infty} \frac{L B c(b, r)}{2 r} \geqq \frac{1}{2} .
$$

12. We shall also use the following property of normal sets.

THEOREM 4. If a set $B$ is normal and $A$ is a linearly measurable subset of $B$ with $L A$ finite, then $A$ is also normal. $\ddagger$

First, any closed subset $K$ of $B$ is normal. Let $F_{K}$ be a family of circles covering $K$, and, since $K$ is closed, $F_{B-K}$ a family of circles none with a point in common with $K$, covering $B-K$. Then $F_{K}$ together with $F_{B-K}$ constitutes a family $F_{B}$ of circles covering $B$. But $B$ is normal, so there exists in $F_{B}$ a sequence of mutually exclusive circles $c_{1}, c_{2}, \ldots$ whose union $s$ contains almost all of $B$. However, those circles $c_{1}^{\prime}, c_{2}^{\prime}, \cdots$ of this sequence having points in common with $K$ are circles of $F_{K}$ and their union $s^{\prime}$ is such that $K-K s^{\prime} \subset B-B s$. Thus $L\left(K-K s^{\prime}\right) \leqq L(B-B s)=0$, so $K$ is normal.

Next $A$ is normal. For let $F_{A}$ be a family of circles covering $A$ and $\epsilon_{n}$ a sequence of decreasing numbers approaching zero. Since $A$ is linearly measurable with $L A$ finite, there exists a closed subset $K_{1}$ of $A$ such that $L A<L K_{1}$ $+\epsilon_{1} / 2$. But $K_{1}$, a closed subset of $B$, is normal, so there exists a finite number of mutually exclusive circles of $F_{A}$ whose union $s_{1}$ is such that $L K_{1}<L K_{1} s_{1}$ $+\epsilon_{1} / 2$. Thus $L A<L K_{1} s_{1}+\epsilon_{1} \leqq L A s_{1}+\epsilon_{1}$. But $s_{1}$ is closed, so the circles of $F_{A}$

$\dagger$ The inequality $r \leqq \Phi_{0} B c(b, r)$, plausible as it seems, has not been established. That caution is necessary with $\Phi_{0}$ measure is indicated by the example of a set $A$ in the ring $c\left(p, r^{\prime}\right)-c(p, r), r^{\prime}>r$, with a point on every radius, but with $\Phi_{0} A=0$ (and of course $L A \geqq 2 \pi r$ ), given by Saks, Fundamenta Mathematicae, vol. 9, p. 16. If, however, the inequality were established, our results would hold for $\Phi_{0}$, as well as for Carathéodory, linear measure.

$\ddagger$ Results similar to those of this theorem and its corollary were indicated by Schauder. However, his theorem VIII does not follow from his previous work since he has not proved the closed subset theorem for the general measure $\Phi$. A theorem that does follow would be obtained if $\Phi$ were replaced by $\Phi_{0}$ throughout. 
with no point in common with $s_{1}$ constitute a family $F_{A-A s_{1}}$ covering $A-A s_{1}$. Hence in $F_{A-A s_{1}}$ there exists a finite number of circles whose union $s_{2}$ is such that $L\left(A-A s_{1}\right)<L A s_{2}+\epsilon_{2}$, or, since $s_{1}$ and $s_{2}$ have no point in common, $L\left[A-A\left(s_{1}+s_{2}\right)\right]<\epsilon_{2}$. Consequently, there exists a sequence of mutually exclusive sets $s_{1}, s_{2}, \cdots$, each the union of a finite number of mutually exclusive circles of $F_{A}$, such that for each $n, L\left[A-A\left(s_{1}+s_{2}+\cdots\right)\right]$ $\leqq L\left[A-A\left(s_{1}+\cdots+s_{n}\right)\right]<\epsilon_{n}$, so $A$ is normal.

Corollary. If $L B$ is finite, $F_{A}$ a family of circles covering $A$, and $\epsilon$ an arbitrary positive number, there exists in $F_{A}$ a sequence of mutually exclusive circles whose union $s$ is such that simultaneously

$$
L A=L A s \text { and } L(B-A) s<\epsilon .
$$

Since the set $B-A$ is linearly measurable with $L(B-A)$ finite, it contains a closed subset $K$ such that $L[(B-A)-K]<\epsilon$. Then the circles of $F_{A}$ without points in common with $K$ again constitute a family $F_{A}{ }^{\prime}$ covering $A$. Thus in $F_{A}{ }^{\prime}$ there exists a sequence of mutually exclusive circles whose union $s$ is such that $L A=L A s$. But the part of $s$ in common with $B-A$ is at most $(B-A)-K$, so $L(B-A) s<\epsilon$.

\section{Projection of a Set AND FURTher nORMality PROPERTIES}

13. Let $B$ be a linearly measurable set with $L B$ finite. From $\$ 5$, the projection $B_{X}$ of $B$ on the $x$-axis is linearly measurable.

We shall show that for a fixed point $p$ the two functions $L B c(p, r)$ and $m[B c(p, r)]_{X}$ of $r$ are continuous from the right. With $r_{n}$ a decreasing sequence approaching $r_{0}>0$, temporarily let $B_{n}=B c\left(p, r_{n}\right)$ and $B_{0}=B c\left(p, r_{0}\right)$. The intersection of the sets $B_{n}$ is $B_{0}$, so $\lim _{n \rightarrow \infty} L B_{n}=L B_{0}$. Also $\left(B_{n}\right)_{X}=\left(B_{0}\right)_{X}$ $+\left(B_{n}-B_{0}\right)_{X}$, so $m\left(B_{n}\right)_{X}-m\left(B_{0}\right)_{X} \leqq m\left(B_{n}-B_{0}\right)_{X} \leqq L\left(B_{n}-B_{0}\right)$, and both functions have right hand continuity in $r$.

Next for $r$ fixed the set of points $P_{\lambda}$ where $L B c(p, r) \geqq \lambda>0$ is closed. For suppose $L B c\left(p_{0}, r\right)<\lambda$ where $p_{0}$ is a limit point of $P_{\lambda}$. We first choose a $\delta>0$ such that $L B c\left(p_{0}, r+\delta\right)<\lambda$, then a point $\bar{p}$ of $P_{\lambda}$ such that $c(\bar{p}, r) \subset c\left(p_{0}, r+\delta\right)$, and thus obtain a contradiction. In the same way the set of points of the plane where $m[B c(p, r)]_{X} \geqq \lambda>0$ is seen to be closed. One then sees that the set of points where these functions take on values between two constants is linearly measurable. Hence, following the terminology of the Lebesgue theory, we say for $r$ fixed $L B c(p, r)$ and $m[B c(p, r)]_{x}$ are linearly measurable functions of $p$.

14. We shall let $B_{0}$ represent the set of all points $p$ of $B$ such that $L B c(p, r)=0$ for some circle. By the Lindelöf-Young theorem $\dagger$ there is a

† Carathéodory, Vorlesungen über Reelle Funktionen, 1927, p. 46. 
sequence of such circles whose union contains $B_{0}$. But the intersection of this union with $B$ has linear measure zero, so $L B_{0}=0$.

Thus for every point $\beta$ of $B-B_{0}$ the quotient

$$
\frac{m[B c(\beta, r)]_{X}}{L B c(\beta, r)}=Q(B, \beta, r)
$$

is defined. Moreover, for $r$ fixed, $Q(B, \beta, r)$ is the quotient of two linearly measurable functions, so is itself linearly measurable on $B-B_{0}$. Also for $\beta$ fixed, $Q(B, \beta, r)$ has right hand continuity in $r$.

If $Q(B, \beta, r)$ has a limit as $r$ approaches zero, we designate this limit by $C(B, \beta)$, otherwise by $\bar{C}(B, \beta)$ and $\underline{C}(B, \beta)$ its limit superior and limit inferior. $\dagger$ We extend these functions to all points of the plane by arbitrarily assigning the value zero at points not in $B-B_{0}$.

The function $\bar{C}(B, \beta)$ is linearly measurable on $B-B_{0}$. For let $r_{n}$ be a decreasing sequence of numbers approaching zero and $q_{1}, q_{2}, \cdots$ the positive rational numbers in some ordering. Then let

$\bar{R}\left(\beta, r_{n}\right)=$ least upper bound of $Q\left(B, \beta, q_{m}\right)$ for $0<q_{m}<r_{n}$, $\bar{F}\left(\beta, r_{n}\right)=$ least upper bound of $Q(B, \beta, r)$ for $0<r<r_{n}$.

Then $\bar{R}\left(\beta, r_{n}\right) \leqq F\left(\beta, r_{n}\right)$. But the right hand continuity in $r$ of $Q(B, \beta, r)$ reveals that $\bar{R}\left(\beta, r_{n}\right) \geqq \bar{F}\left(\beta, r_{n}\right)$, so these two functions are equal. Both functions are then linearly measurable on $B-B_{0}$, since $\bar{R}\left(\beta, r_{n}\right)$ is the least upper bound of a sequence of functions linearly measurable on $B-B_{0}$. But it is seen that

$$
\bar{C}(B, \beta)=\limsup _{r \rightarrow 0} Q(B, \beta, r)=\lim _{n \rightarrow \infty} F\left(\beta, r_{n}\right) .
$$

Thus $\bar{C}(B, \beta)$ is the limit of a sequence of functions linearly measurable on $B-B_{0}$, so is itself linearly measurable on $B-B_{0}$.

A similar procedure shows that $\underline{C}(B, \beta)$ is also linearly measurable on $B-B_{0}$. Consequently $\bar{C}(B, p)$ and $\underline{C}(B, p)$ are linearly measurable on the plane or on any linearly measurable subset $E$ of the plane. But these functions are bounded, so if $L E$ is finite, the integrals $\int_{E} \bar{C}(B, p) d L$ and $\int_{E} \underline{C}(B, p) d L$, taken over $E$ in the sense of Lebesgue with respect to Carathéodory linear measure, exist.

15. We now let $B$ be normal, in addition to being linearly measurable with $L B$ finite, and prove two lemmas and an important integral theorem.

LEMMA 1. If $A$ is a linearly measurable subset of $B$ that projects on the $x$-axis in a set of Lebesgue measure zero, then $\bar{C}(B, \alpha)=0$ at almost all points $\alpha$ of $A$.

$\dagger$ While no notion of direction is involved here, yet if $\beta$ were a point of an ordinary curve, $C(B, \beta)$ would be the absolute value of the cosine of the angle between the $x$-axis and the direction of $B$ at $\beta$. 
Since $\bar{C}(B, p)$ is a linearly measurable function, the subset $A_{\lambda}$ of $A$ where $\bar{C}(B, p)>\lambda>0$ is linearly measurable, and hence, being a subset of a normal set $B$, is normal. Suppose the lemma is not true. There is then a $\lambda>0$ such that also $L A_{\lambda}>0$. Then the collection of all circles $c(\alpha, r)$, with centers at points of $A_{\lambda}$, such that

$$
\frac{\lambda}{2}<\frac{m[B c(\alpha, r)]_{x}}{L B c(\alpha, r)}
$$

is a family $F_{A_{\lambda}}$ of circles covering $A_{\lambda}$.

Since $A_{\lambda}$ projects on the $x$-axis in a set of Lebesgue measure zero,

$$
m[B c(\alpha, r)]_{X}=m\left[\left(B-A_{\lambda}\right) c(\alpha, r)\right]_{X} \leqq L\left(B-A_{\lambda}\right) c(\alpha, r) .
$$

Also $L A_{\lambda} c(\alpha, r) \leqq L B c(\alpha, r)$. Thus for each circle of $F_{A_{\lambda}}$, from (1),

$$
\frac{\lambda}{2} L A_{\lambda} c(\alpha, r) \leqq L\left(B-A_{\lambda}\right) c(\alpha, r) .
$$

But, with $\epsilon$ an arbitrary positive number, from the corollary of $\$ 12$, there exists a sequence of mutually exclusive circles of $F_{A_{\lambda}}$ such that for their union $s, L A_{\lambda} s=L A_{\lambda}$ and $L\left(B-A_{\lambda}\right) s<\epsilon$. We thus have the contradiction, $(\lambda / 2) L A_{\lambda}<\epsilon$, to our assumption.

LEMMA 2. Let $A$ be a linearly measurable subset of $B$ with at most one point on each line perpendicular to the $x$-axis. If at each point $\alpha$ of $A$

$$
\lambda \leqq \dot{C}(B, \alpha) \text {, then } \lambda L A \leqq m A_{X},
$$

or if

$$
\lambda>\dot{C}(B, \alpha) \text {, then } \lambda L A \geqq m A_{X},
$$

where $\dot{C}(B, \alpha)$ means either $\bar{C}(B, \alpha)$ or $\underline{C}(B, \alpha)$.

We shall prove only the first part of this lemma.

Let $\eta$ be an arbitrary positive number. For either interpretation of $\dot{C}(B, \alpha)$, the collection of all circles $c(\alpha, r)$ with centers at points of $A$ for which the inequality

$$
\lambda-\eta<\frac{m[B c(\alpha, r)]_{X}}{L B c(\alpha, r)}
$$

holds, is a family $F_{A}$ of circles covering $A$. But $A$ is a subset of $B$, so for each circle of $F_{A},(\lambda-\eta) L A c(\alpha, r)<m[B c(\alpha, r)]_{X} \leqq m[A c(\alpha, r)]_{X}+m[(B-A) c(\alpha, r)]_{X}$ $\leqq m[A c(\alpha, r)]_{x}+L(B-A) c(\alpha, r)$.

Since there is at most one point of $A$ on any line perpendicular to the $x$-axis, mutually exclusive subsets of $A$ project into mutually exclusive sets. 
Thus if $s$ is the union of any sequence of mutually exclusive circles of $F_{A}$, $(\lambda-\eta) L A s<m(A s)_{X}+L(B-A) s$. But (corollary $\left.\$ 12\right)$ with $\epsilon>0$ arbitrary there is an $s$ such that $L A s=L A$ and $L(B-A) s<\epsilon$. For this $s$ also $m(A s)_{X}=m A_{X}$, so we have $(\lambda-\eta) L A<m A_{X}+\epsilon$. Thus $\lambda L A \leqq m A_{X}$.

Now let $E$ be a linearly measurable subset of $B$ with at most one point on each perpendicular to the $x$-axis. From Lemma 2 we see that

$$
m E_{X}=\int_{E} \bar{C}(B, p) d L=\int_{E} \underline{C}(B, p) d L .
$$

But $\bar{C}(B, p) \geqq \underline{C}(B, p)$ so $C(B, p)$ exists at almost all points of $E$.

Furthermore, $C(B, p)$ exists at almost all points of $B$. For there is a sequence of closed subsets of $B$ whose union $\underline{B}$ has $L \underline{B}=L B$. But (Theorems 1 and 2) $\underline{B}$ is the union of a sequence of linearly measurable sets $B^{1}, B^{2}, \ldots$ (each with at most one point on any line perpendicular to the $x$-axis) and a linearly measurable set $\mathcal{B}^{\infty}$ (every point of whose projection is the image of an infinite number of points of $\underline{B}$ ). From consideration of the above integrals, $C(B, p)$ exists at almost all points of each set $B^{m}$, and consequently at almost all points of the union $\sum_{m=1}^{\infty} B^{m}$. Also, from $\S 5, m\left(\mathcal{B}^{\infty}\right)_{X}=0$, so, Lemma 1 , $C(B, p)$ exists and is zero at almost all points of $\mathbb{B}^{\infty}$. Thus $C(B, p)$ exists at almost all points of $\underline{B}$ so finally at almost all points of $B$.

We later make direct use of

TheORem 5. Let $E$ be a linearly measurable subset of $B$ with at most one point on any line $x=x_{0}$, and let $F(p)$ be a function summable on $B$ with respect to Carathéodory linear measure. Then the function of $x$ defined for each value $x_{0}$ as

$$
f\left(x_{0}\right)=\left\{\begin{array}{l}
0 \text { if } x_{0} \text { is not } a \text { point of } E_{X}, \text { otherwise } \\
F(p), \text { where } p \text { is the point of } E \text { on the line } x=x_{0},
\end{array}\right.
$$

is summable on $E_{X}$ with respect to Lebesgue linear measure and

$$
\int_{E_{X}} f(x) d x=\int_{E} F(p) \bar{C}(B, p) d L
$$

First $F(p) \bar{C}(B, p)$ is linearly measurable on $E$, since it is the product of two such functions. Then $f(x)$ is Lebesgue measurable on $E_{X}$, since the part of $E_{X}$ where $f(x)>k$ is the projection of the linearly measurable subset of $E$ where $F(p)>k$.

With $M$ and $N$ two non-negative numbers, define

$$
F_{M N}(p)=\{-M \text { if } F(p)<-M, F(p) \text { if }-M \leqq F(p)<N, N \text { if } F(p) \geqq N\}
$$


and in a like manner, $f_{M N}(x)$. Let $-M=a_{0}, a_{1}, \cdots, a_{n}=N$ be a subdivision of the interval $(-M, N)$. Call $E_{k}$ the subset of $E$ where $a_{k-1} \leqq F_{M N}(p)<a_{k}$. Then $\left(E_{k}\right)_{X}$ is the subset of $E_{X}$ where $a_{k-1} \leqq f_{M N}(x)<a_{k}$.

Consequently, from Lemma 2,

$$
m\left(E_{k}\right)_{X}=\int_{E_{k}} \bar{C}(B, p) d L .
$$

Then

so

$$
a_{k-1} m\left(E_{k}\right)_{X} \leqq \int_{E_{k}} F_{M N}(p) \bar{C}(B, p) d L \leqq a_{k} m\left(E_{k}\right)_{X}
$$

$$
\sum_{k=1}^{n} a_{k-1} m\left(E_{k}\right)_{X} \leqq \int_{E} F_{M N}(p) \bar{C}(B, p) d L \leqq \sum_{k=1}^{n} a_{k} m\left(E_{k}\right)_{X} .
$$

But this is true of every subdivision of $(-M, N)$, so

$$
\int_{E_{\mathbf{X}}} f_{M N}(x) d x=\int_{\boldsymbol{E}} F_{M N}(p) \bar{C}(B, p) d L .
$$

However, $\int_{E} F(p) \bar{C}(B, p) d L$ exists since the integrand is the product of two functions summable on $E$, one of which is bounded. Consequently, the summability of $f(x)$ and the equality

$$
\int_{E_{\mathbf{X}}} f(x) d x=\int_{\boldsymbol{E}} F(p) \bar{C}(B, p) d L
$$

follows.

\section{A REPLACEMENT FOR DIRECTION AND GENERALIZATION OF THE GAUSS-GREEN LEMMA}

16. We divide the boundary $B$ of a plane set $G$ into three mutually exclusive subsets $B_{\mathrm{I}}, B_{\mathrm{II}}, B_{\mathrm{III}}$; with $B_{\mathrm{I}}$ consisting of two parts. Let $b$ be a point of $B$ and $Y$ the line through $b$ perpendicular to the $x$-axis. Then $b$ shall belong to

$B_{\mathrm{I}_{1}}$ if there is a segment $a \leqq y<b$ of $Y$ below $b$ of points of the complement of $G$ and a segment $b<y \leqq c$ of $Y$ above $b$ of points of $G$.

$B_{\mathrm{I}_{2}}$ if there is a segment $a \leqq y<b$ of points of $G$ and a segment $b<y \leqq c$ of points of the complement of $G$.

$B_{\mathrm{II}}$ if there exist two segments $a \leqq y<b$ and $b<y \leqq c$ which either both contain only points of $G$, or both contain only points of the complement of G.

$B_{\text {III }}$ if either half of every segment of $Y$ with mid point $b$ contains both points of $G$ and points of the complement of $G$. 
One sees that a line perpendicular to the $x$-axis through a point of $B_{\text {III }}$ contains an infinite number of points of $B$, i.e., $B_{\mathrm{III}} \subset \mathbb{B}^{\infty}$.

17. In the introduction we pointed out that Schauder made the material restriction that $m\left(B_{\mathrm{II}}\right)_{X}=0$. We are able to avoid this restriction by showing eventually that $B_{\mathrm{II}}$, and also $\mathcal{B}^{\infty}$, contribute nothing to the boundary integral. The result is then obtained by integrating over the remaining part of $B$, i.e., over $B_{\mathrm{I}}-B_{\mathrm{I}} B^{\infty}$. However, we find it convenient to integrate over $B_{\mathrm{I}_{1}}$ $-B_{\mathrm{I}_{1}} B^{\infty}$ and $B_{\mathrm{I}_{2}}-B_{\mathrm{I}_{2}} \mathcal{B}^{\infty}$ separately. It is thus necessary to know that these two sets are linearly measurable. We give the demonstration only for the first set.

First let $B_{\mathrm{I}_{1} n}$ be all points $b$ of $B$ such that $b-1 / n \leqq y<b$ consists only of points of the complement of $G$ while $b<y \leqq b+1 / n$ consists only of points of $G$. Let $b_{0}$ be a limit point of $B_{\mathrm{I}_{1} n}$. Then $b_{0}$ is a point of $B$, since $B$ is closed. If $b_{0}$ is a point of $B_{\mathrm{I}_{2}}$, there is an interval $a \leqq y<b$, of points of $G$, each of which is a limit point of points of the complement of $G$, i.e., $a \leqq y \leqq b$ is an interval of boundary points, so $b_{0}$ belongs to $B^{\infty}$. In a like manner one sees that each limit point of $B_{\mathrm{I}_{1} n}$ which is a point of $B_{\mathrm{II}}$ is a point of $\mathscr{B}^{\infty}$.

Hence with $\bar{B}_{\mathrm{I}_{1} n}$ the closure of $B_{\mathrm{I}_{1} n}, \bar{B}_{\mathrm{I}_{1} n} \subset B_{\mathrm{I}_{1}}+\mathcal{B}^{\infty}$ and $\sum_{n=1}^{\infty} \bar{B}_{\mathrm{I}_{1} n} \supset B_{\mathrm{I}_{1}}$, so $\sum_{n=1}^{\infty}\left(\bar{B}_{\mathrm{I}_{1} n}+\mathcal{B}^{\infty}\right)=B_{\mathrm{I}_{1}}+\mathcal{B}^{\infty}$. Thus $B_{\mathrm{I}_{1}}+\mathcal{B}^{\infty}$ is linearly measurable because each $\bar{B}_{\mathrm{I}_{1} n}$ is linearly measurable (closed) and $\mathscr{B}^{\infty}$ is linearly measurable (Theorem 2). Consequently, the set $B_{\mathrm{I}_{1}}-B_{\mathrm{I}_{1}} B^{\infty}$ is linearly measurable since it is the intersection $\left(B-B^{\infty}\right)\left(B_{\mathbf{I}_{1}}+\mathcal{B}^{\infty}\right)$ of two linearly measurable sets.

Likewise, the set $B_{\mathrm{I}_{2}}-B_{\mathrm{I}_{2}} \mathcal{B}^{\infty}$ is linearly measurable. Thus $B_{\mathrm{I}}-B_{\mathrm{I}} \mathcal{B}^{\infty}$, and then $B_{\mathrm{II}}-B_{\mathrm{II}} \mathrm{B}^{\infty}$, is linearly measurable.

18. At all points $b$ of $B$ we now define the function

$$
\cos (B, b)=\left\{\begin{array}{l}
-\bar{C}(B, b) \text { if } b \text { is a point of } B_{\mathrm{I}_{1}}-B_{\mathrm{I}_{1}} B^{\infty} \\
\bar{C}(B, b) \text { if } b \text { is a point of } B_{\mathrm{I}_{2}}-B_{\mathrm{I}_{2}} B^{\infty} \\
0 \text { if } b \text { is a point of } B_{\mathrm{II}}-B_{\mathrm{II}} B^{\infty} \\
\bar{C}(B, b) \text { if } b \text { is a point of } \mathcal{B}^{\infty} .
\end{array}\right.
$$

If $b$ is given by its coordinates $(x, y)$ we designate

$$
\cos (B, b) \text { by } \cos [B,(x, y)] \text {. }
$$

Since, as we have seen, $C(B, b)$ exists at almost all points of $B$ it would have been as well, in the following integral theorems, to define $\cos (B, b)$ in terms of $C(B, b)$. Also, since $\bar{C}(B, b)=0$ at almost all points of $\mathcal{B}^{\infty}$, we could have defined $\cos (B, b)=0$ at all points of $B^{\infty}$.

In the ordinary Green's theorem, the points of a crosscut correspond essentially to $B_{\mathrm{II}}-B_{\mathrm{II}} \mathrm{B}^{\infty}$. Since the integral around the complete boundary traverses a crosscut twice, and its value when taken in one direction is annulled by its value in the opposite direction, the same result would be ob- 
tained if at each point of a crosscut the direction cosine of the external normal were arbitrarily replaced by zero. In an analogous manner, even though there is no notion of direction in our case, we have defined $\cos (B, b)$ to be zero at all points of $B_{\mathrm{II}}-B_{\mathrm{II}} \mathrm{B}^{\infty}$.

19. We now prove the theorem connecting a double integral over a plane set and a single integral over the boundary of the set.

THEOREM 6. Let $G$ be a bounded plane point set whose boundary $B$ is normal with $L B$ finite, and let $(G+B) x_{0}$ be the intersection of $G+B$ and the line $x=x_{0}$. If a function $F(x, y)$ is summable on $B$ with respect to linear measure and is absolutely continuous in $y$ on $(G+B) x_{0}$ for almost all values of $x_{0}$ in $B_{X}$, and if $\partial F(x, y) / \partial y$ is summable on $G+B$ with respect to Lebesgue plane measure, then $F(x, y) \cos [B,(x, y)]$ is summable on $B$ with respect to linear measure, and

$$
\iint_{G} \frac{\partial F(x, y)}{\partial y} d x d y=\int_{B} F(x, y) \cos [B,(x, y)] d L .
$$

The plane measure of $B$ is zero since $L B$ is finite, $\nmid$ hence

$$
\iint_{G} \frac{\partial F(x, y)}{\partial y} d x d y=\iint_{G+B} \frac{\partial F(x, y)}{\partial y} d x d y=\int_{B_{X}} d x \int_{(G+B) x} \frac{\partial F(x, y)}{\partial y} d y .
$$

But the set $\mathscr{B}^{m}$ (of all points of $B$ on all lines perpendicular to the $x$-axis containing exactly $m$ points of $B$ ) is linearly measurable, so

$$
\begin{aligned}
\int_{B_{X}} d x \int_{(G+B) x} \frac{\partial F(x, y)}{\partial y} d y= & \int_{\left(\mathcal{B}^{\infty}\right)_{X}} d x \int_{(G+B) x} \frac{\partial F(x, y)}{\partial y} d y \\
& +\sum_{m=1}^{\infty} \int_{\left(\mathcal{B}^{m}\right)_{X}} d x \int_{(G+B) x} \frac{\partial F(x, y)}{\partial y} d y .
\end{aligned}
$$

However, since $m\left(\mathcal{B}^{\infty}\right)_{X}=0$ and $\bar{C}[B,(x, y)]=0$ at almost all points of $\mathcal{B}^{\infty}$,

$$
\int_{\left(\mathcal{B}^{\infty}\right)_{X}} d x \int_{(G+B) x} \frac{\partial F(x, y)}{\partial y} d y=\int_{\mathcal{B}^{\infty}} F(x, y) \cos [B,(x, y)] d L=0 .
$$

Thus the proof of the theorem will be complete upon showing that $F(x, y)$ $\cdot \cos [B,(x, y)]$ is summable on $B^{m}$ and

(A) $\int_{\left(\mathcal{B}^{m}\right)_{X}} d x \int_{(G+B) x} \frac{\partial F(x, y)}{\partial y} d y=\int_{\mathcal{B}^{m}} F(x, y) \cos [B,(x, y)] d L$.

Toward proving (A), let $x_{0}$ be a point of $\left(\mathcal{B}^{m}\right)_{X}$. Then each of the $m$ points of $B$ on $x=x_{0}$ belongs to either $B_{\mathrm{I}}$ or $B_{\mathrm{II}}$. If there are points of $B_{\mathrm{I}}$ on

† Göttinger Nachrichten, 1914, p. 425. 
$x=x_{0}$, there are an even number of such points; the lowest belonging to $B_{\mathrm{I}_{1}}$, the highest to $B_{\mathrm{I}_{2}}$. With $\left(x_{0}, b_{1}\right),\left(x_{0}, b_{2}\right), \cdots,\left(x_{0}, b_{2 n}\right)$ the points of $B_{\mathrm{I}}$ in order of increasing ordinates, the set $(G+B) x_{0}$ consists of the non-abutting intervals $b_{2 i-1} \leqq y \leqq b_{2 i}, i=1,2, \cdots, n$, and a finite number of points of $B_{\text {II }}$. Thus

$$
\int_{(G+B) x_{0}} \frac{\partial F\left(x_{0}, y\right)}{\partial y} d y=\sum_{i=1}^{n} \int_{b_{2 i-1}}^{b_{2 i}} \frac{\partial F\left(x_{0}, y\right)}{\partial y} d y=\sum_{i=1}^{2 n}(-1)^{i} F\left(x_{0}, b_{i}\right),
$$

except perhaps for a set of measure zero made up of the values of $x_{0}$ where the integral on the left fails to exist and those values of $x_{0}$ for which $F\left(x_{0}, y\right)$ is not absolutely continuous. The second equality follows from the fact that an absolutely continuous function is reproduced by the integral of its derivative. $\dagger$

We recall that the set $B^{r}$ consists of all points $p$ of $B$ such that exactly $r-1$ points of $B$ lie below $p$ on the same perpendicular to the $x$-axis. With $\left(x_{0}, y\right)$ the point of $B^{r}$ on the line $x=x_{0}$, define

$$
f_{r}\left(x_{0}\right) \quad(r=1,2, \cdots, m)
$$

as $-F\left(x_{0}, y\right), F\left(x_{0}, y\right)$ or zero according as $\left(x_{0}, y\right)$ belongs to $B_{\mathrm{I}_{1}}, B_{\mathrm{I}_{2}}$, or $B_{\mathrm{II}}$. Then since $\left(x_{0}, b_{i}\right)$ belongs to $B_{\mathrm{I}_{1}}$ if $i$ is odd or to $B_{\mathrm{I}_{2}}$ if $i$ is even,

$$
\sum_{r=1}^{m} f_{r}\left(x_{0}\right)=\sum_{i=1}^{2 n}(-1)^{i} F\left(x_{0}, b_{i}\right) \text {. }
$$

Consequently, from (1), $\sum_{r=1}^{m} f_{r}(x)$ is summable on $\left(\mathcal{B}^{m}\right)_{X}$ and

$$
\int_{\left(\mathcal{B}^{m}\right)_{X}} d x \int_{(G+B) x} \frac{\partial F(x, y)}{\partial y} d y=\int_{\left(\mathcal{B}^{m}\right)_{X}} \sum_{r=1}^{m} f_{r}(x) d x .
$$

Since $F(x, y)$ is summable on $B$ and $B^{r}$ has at most one point on any line perpendicular to the $x$-axis, while all three sets $B_{\mathrm{I}_{1}}-B_{\mathrm{I}_{1}} B^{\infty}, \mathcal{B}^{\infty}$, and $B^{r}$ are linearly measurable, it follows from Theorem 5 and the definition of $\cos [B,(x, y)]$ at points of $B_{\mathrm{I}_{1}}$ that

$$
\begin{aligned}
\int_{\left(B_{\mathrm{I}_{1}} \mathcal{B}^{m} B^{r}\right)_{x}} f_{r}(x) d x & =\int_{B_{\mathrm{I}_{1}} \mathcal{B}^{m} B^{r}}-F(x, y) \bar{C}[B,(x, y)] d L \\
& =\int_{B_{\mathrm{I}_{1}} \mathcal{B}^{m} B^{r}} F(x, y) \cos [B,(x, y)] d L .
\end{aligned}
$$

In like manner

$$
\int_{\left(B_{\mathbb{1}_{2}} \mathcal{B}^{m} B^{r}\right)_{\mathbf{X}}} f_{r}(x) d x=\int_{B_{\mathrm{I}_{2}} \mathcal{B}^{m} B^{r}} F(x, y) \cos [B,(x, y)] d L .
$$

† Hobson, Real Variables, p. 553. 
But $f_{r}(x)=0$ at each point of $\left(B_{\mathrm{II}} B^{r}\right)_{X}$ and $\cos [B,(x, y)]=0$ at each point of $B_{\mathrm{II}}-B_{\mathrm{II}} \mathrm{B}^{\infty}$, so

$$
\int_{\left(B_{\mathrm{II}} \mathcal{B}^{m} B^{r}\right)_{X}} f_{r}(x) d x=\int_{B_{\mathrm{II}} \mathcal{B}^{m} B^{r}} F(x, y) \cos [B,(x, y)] d L=0 .
$$

Consequently, from the last three equations, by addition,

$$
\int_{\left(\mathcal{B}^{m}\right)_{x}} f_{r}(x) d x=\int_{\mathcal{B}^{m} B^{r}} F(x, y) \cos [B,(x, y)] d L .
$$

Now, since $f_{r}(x)$ is summable on $\left(B^{m}\right)_{X}$, we have

$$
\int_{\left(\mathcal{B}^{m}\right)_{X}} \sum_{r=1}^{m} f_{r}(x) d x=\sum_{r=1}^{m} \int_{\left(\mathcal{B}^{m}\right)_{X}} f_{r}(x) d x .
$$

But from the last two equations and (2)

$$
\begin{aligned}
\int_{\left(\mathcal{L}^{m}\right)_{X}} d x \int_{(G+B) x} \frac{\partial F(x, y)}{\partial y} d y & =\sum_{r=1}^{m} \int_{\mathcal{B}^{m} B^{r}} F(x, y) \cos [B,(x, y)] d L \\
& =\int_{\mathcal{B}^{m}} F(x, y) \cos [B,(x, y)] d L
\end{aligned}
$$

which is equation (A). The proof is thus complete.

In Theorem 6 the only condition on the boundary $B$ of $G$ is that it be normal and have $L B$ finite, while there is no condition on $G$ except that it be bounded. The boundedness of $G$ and the finiteness of $L B$ are inherent in the problem, whereas the normality of $B$ is a less natural restriction introduced to fit the method of attack. That, however, the normality condition is not a very drastic restriction is shown by the fact that the boundary of every simply connected region is normal if this boundary has Carathéodory linear measure finite. Furthermore, it follows that a much larger class of sets $G$ have normal boundaries. For let $G$ be a set whose boundary $B$ is contained in the union $B^{\prime}$ of the boundaries $B_{1}, B_{2}, \cdots, B_{n}$ of a finite number of simply connected regions, where each $B_{i}$ has Carathéodory linear measure finite. Then $B^{\prime}$ is normal (lemma $\$ 10$ ) since $\underline{D}\left(B^{\prime}, b\right) \geqq 1 / 2$ at each point $b$ of $B^{\prime}$. Consequently, $B$ is normal (Theorem 4 ) since it is a linearly measurable subset of $B^{\prime}$. The usual crosscut scheme is included in this extension.

CoRnell UnIVERsity, ITHACA, N. Y. 